\title{
RESEARCH
}

Open Access

\section{Platelet-activating factors are associated with cognitive deficits in depressed coronary artery disease patients: a hypothesis-generating study}

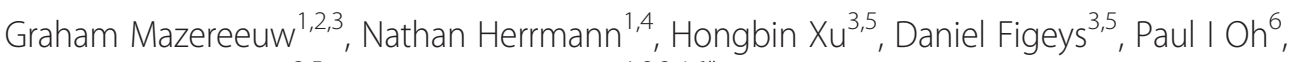

Steffany AL Bennett ${ }^{3,5}$ and Krista L Lanctôt $\hat{t}^{1,2,3,4,6^{*}}$

\begin{abstract}
Background: Patients with coronary artery disease (CAD) are at risk of accelerated cognitive decline, particularly those with major depression. Mechanisms for cognitive deficits associated with CAD, and the effects of depression, remain poorly understood. However, CAD is associated with inflammatory processes that have been linked to neurodegeneration, may contribute to cognitive decline, and are elevated in depression. Platelet-activating factors (PAFs) are emerging as key lipid mediators that may be central to those processes and highly relevant to cognitive decline in CAD.

Methods: This cross-sectional study investigated relationships between various PAFs and cognitive performance in 24 patients with CAD (age, $60.3 \pm 9.4 ; 70.8 \%$ male). Analyses were repeated in a subgroup of 15 patients with CAD with major depression (DSM-IV). Cognitive performance was assessed using a standardized battery and summary $z$ scores were calculated based on age, sex, and education norms. Global cognitive performance was the average of domain-specific $z$ scores. Plasma PAF analyses were performed using electrospray ionization mass spectrometry (precursor ion scan).

Results: A greater abundance of PAF PC(O-18:0/2:0) was associated with poorer global cognitive performance in patients with CAD $(r=-0.45, P=0.03)$. In the major depressed subgroup, PAF PC(O-18:0/2:0) $(r=-0.59, P=0.02)$ as well as $P C(0-16: 0 / 2: 0)(r=-0.52, P=0.04)$, and lyso-PAF PC(O-16:0/0:0) $(r=-0.53, P=0.04)$ were associated with poorer global cognitive performance. A greater abundance of PAF PC(O-19:5/2:0) was associated with better global cognitive performance $(r=0.55, P=0.03)$, suggesting a possible compensatory species.

Conclusions: This study suggests that certain PAFs might be associated with global cognitive performance in patients with CAD, with stronger relationships observed in those with major depression. Confirmation of these preliminary findings is warranted.
\end{abstract}

Keywords: Biomarker, cognition, coronary artery disease, depression, executive function, inflammation, lipidomics, memory, neurodegeneration, platelet-activating factor

\footnotetext{
* Correspondence: Krista.Lanctot@sunnybrook.ca

'Sunnybrook Research Institute, Sunnybrook Health Sciences Centre,

2075 Bayview Avenue, Room FG08, Toronto, ON M4N 3M5, Canada

${ }^{2}$ Department of Pharmacology and Toxicology, University of Toronto,

Toronto, ON, Canada

Full list of author information is available at the end of the article
} 


\section{Background}

Cognitive deficits are prevalent in patients with coronary artery disease (CAD) [1-4] and, accordingly, patients with CAD are at risk of accelerative cognitive decline and transition to dementia [5]. Major depression significantly amplifies the rate of cognitive decline in those with CAD [6,7], as well as worsening functional outcomes and increasing the risk of mortality $[8,9]$. The prevention of cognitive decline in patients with CAD may rely not only on adequate treatment of depressive symptoms, but also on a targeted inhibition of the mechanisms leading to cognitive decline in those with depression. Unfortunately, response rates to antidepressant therapies are modest in patients with CAD $[10,11]$ and there are no consistently effective treatments to prevent cognitive decline [12]. Furthermore, the mechanisms of cognitive decline in patients with CAD, and the contributions of depression, are poorly understood.

Recent reviews have implicated aberrant lipid metabolism, inflammatory activity, and oxidative stress as pathophysiological processes involved in cognitive decline $[13,14]$ and these same mechanisms have been associated with depression in CAD $[15,16]$. Persistent activation of these pathways appears to precede neurodegeneration and may contribute to cognitive vulnerability in those with depression [17]. Platelet-activating factors (PAFs) are a diverse family of potent alkylacylglycerophosphocholine (lipid) second messengers. Emerging evidence suggests that PAF activation is a hallmark of pathological disruption to membrane-based lipid signaling cascades regulating inflammatory and apoptotic activity, with various PAFs demonstrating selective pro- and anti-inflammatory and neurodegenerative effects $[13,18]$. Accordingly, PAF activation has been associated with the presence of CAD and with inflammation, oxidative stress, and neurodegeneration; all contemporary mechanisms for depression in CAD [19]. Furthermore, PAFs are increasingly being recognized as key participants in the neurodegenerative mechanisms associated with cognitive decline, with indications of variable effects based on species identity [13,20]. As such, PAFs may be pertinent biomarkers as well as potential mediators of cognitive decline in patients with $\mathrm{CAD}$, with particular relevance to those with major depression. Despite these mechanistic links, the relationships between PAFs and cognitive performance in CAD patients with or without depression have yet to be investigated. Here, we explore a crosssectional association between measures of cognitive performance and the plasma abundance of various PAF species for the first time using a targeted lipidomic approach in patients with CAD, with and without major depression.

\section{Methods}

\section{Ethics statement}

This study was approved by the research ethics boards of Sunnybrook Research Institute, University Health Network at Toronto Rehabilitation Institute, and Trillium Health Partners, and was conducted according to the principles expressed in the Declaration of Helsinki.

\section{Participants}

Participants were approached at entry into a cardiac rehabilitation program at two local centers, the UHN Toronto Rehabilitation and Trillium Health Partners cardiac centers in Toronto, Ontario between September 2010 and May 2012. Inclusion criteria consisted of the presence of CAD (myocardial infarction, percutaneous transluminal coronary angioplasty, coronary artery bypass graft, or angiographic evidence of at least 50\% stenosis in one or more major coronary arteries) with a minimum of 7 weeks post-intervention, to ensure CAD stability. Participants aged 45 to 80 , who spoke and understood English, and provided written and informed consent were considered. Once included, demographic information, a detailed medical history, and concomitant medications were recorded. The use of antidepressant medication was permitted on the condition of dose stability over at least 3 months, to minimize potential confounding effects (psychological or physiological) of adjustment to these medications on the study findings. Participants were excluded if they had a history of a neurological condition, had a pre-morbid diagnosis of any Axis I psychiatric disorder other than depression, or had a significant acute medical illness (for example autoimmune condition, uncontrolled diabetes mellitus, or uncontrolled hypothyroidism).

\section{Schedule of assessments}

Performance in specific cognitive domains was assessed using a multi-domain battery specified by the National Institute for Neurological Disorders and Stroke - Canadian Stroke Network guidelines for the study of vascular cognitive impairment [21]. This battery included tests of executive function (the Animal Naming Task, the FAS Controlled Oral Word Association Test, and the Trail Making Test Part B), memory (the California Verbal Learning Test II, immediate and delayed recall, and the Brief Visuospatial Memory Test, immediate and delayed recall), and attention and processing speed (the Digit-Symbol Substitution Test and the Trail Making Test Part A). Scores for each cognitive test were converted to a $z$ score based on age, sex, education, and handedness (where applicable) norms from a representative cognitively healthy population for each test and combined for each domain into a domain composite $z$ score $[21,22]$. Global cognition was taken as the composite $z$ score of all domains.

Depressive symptom severity was measured using the 17-item Hamilton Depression Rating Scale [23] and controlled as a covariate. Patients were also examined for the presence of a major depressive episode by trained study personnel using the Structured Clinical Interview 
for DSM-IV Disorders - Depression scale according to the Diagnostic and Statistical Manual of Mental Disorders (DSM) IV [24] and those meeting the inclusion criteria were included in a subgroup analysis, of the PAF-cognition relationship with $\mathrm{CAD}$ patients with major depression.

\section{Sample collection and lipidomic analyses: lipid extraction and high-performance liquid chromatography electrospray ionization mass spectrometry}

Fasting blood samples were collected at the study visit and extracted plasma was frozen at $-80^{\circ} \mathrm{C}$ and batched for analysis. Plasma lipids were extracted using a modified Bligh and Dyer method [25] adapted, as previously described, for efficient extraction of alkylacylglycerophosphocholines (PAFs) [20,26,27]. The PAF species were analyzed using precursor ion scanning with the diagnosis fragment ion of phosphocholine head group at $m / z 184$, as described [20,27]. Identities were predicted for each $m / z$ value using the online phospholipid prediction tool VaLID version 1.1 [28], and validated by analysis of mass spectrometry spectral fragmentation patterns [29] and linear regression models defining retention times of each PAF subgroup, as described previously [20].

\section{Statistics}

The 'peak area' of each lipid, representing the area under the curve in counts per second, was established using Analyst version 1.4.2 (AB Sciex), normalized to the synthetic internal standard $\left(\mathrm{PA}_{\mathrm{PC}(13: 0 / 0: 0)}\right)$ spiked at time of extraction and multiplied by $1 \mathrm{E} 6$ before logarithmic transformation (base 2). The associations between PAF abundance and $z$ scores from cognitive performance tests were investigated using linear regression (Pearson correlation coefficient $(r)$ ) (SPSS statistical software, version 13.0, Chicago, IL, USA). Significant associations between a PAF and global cognitive performance were then tested in independent covariate-adjusted models, controlling for the potential influence of age, sex, years of education, and depressive symptom severity. Participant characteristics associated with global cognitive performance in linear regression or group-comparison analyses were included in independent covariate-adjusted models as needed. As this study is intended to be hypothesis generating, the findings were not adjusted for multiple comparisons.

\section{Results}

Ninety-five patients with CAD were assessed for study eligibility at entry to their cardiac rehabilitation program. Of those, 57 patients were excluded either because they did not agree to participate in the research $(N=41)$ or because they had begun or changed their dose of antidepressant treatment within the previous 3 months $(N=$ 16). Of the remaining 38 eligible patients, 24 agreed to participate in this study (participant characteristics are summarized in Table 1). Those patients included had HAM-D scores ranging from 3 to 20 , and $62.5 \%$ of the patients met DSM-IV criteria for major depression.

\section{Cognitive performance}

Performance across all cognitive tests in this sample of patients was below comparable population norms, with mean $z$ scores ranging from -0.22 to -0.55 (Table 2). Similarly, global cognition, as measured by composite $z$ scores from the tested cognitive domains, was also below norms, with a mean $z$ score of $-0.38( \pm 0.62)$. Cognitive performance was poorer in the major depressed subgroup (Table 2).

\section{Platelet-activating factors and cognitive performance}

Seventy-two phosphocholine second messengers with $\mathrm{m} / \mathrm{z}$ between 450 and 600 were detected in patient plasma samples. Of these, 23 species were identified as PAFs. A significant association between a greater plasma abundance of the PAF PC(O-18:0/2:0) and poorer global cognitive function (composite $z$ score) was observed in the total sample of patients (Table 3). That association appeared to be driven by a significant relationship between a greater plasma abundance of $\mathrm{PC}(\mathrm{O}-18: 0 / 2: 0)$ and poorer executive function $(r=-0.51, P=0.01)$. Trends between a greater plasma abundance of the PAFs $\mathrm{PC}(O-16: 0 / 2: 0), \mathrm{PC}(O-$ $18: 1 / 2: 0)$, and $\mathrm{PC}(O-18: 6 / 2: 0)$ and poorer global cognitive performance were detected (Table 3 ).

The PAF PC(O-18:0/2:0) remained significantly associated with poorer global cognitive performance after adjusting for age $\left(\beta_{\mathrm{PAF}}=-0.45, P=0.03\right)$, sex $\left(\beta_{\mathrm{PAF}}=-0.45, P=\right.$ $0.03)$, years of education $\left(\beta_{\mathrm{PAF}}=-0.46, P=0.03\right)$, and depressive symptom severity $\left(\beta_{\mathrm{PAF}}=-0.46, P=0.03\right)$ in independent models. None of the participant characteristics was significantly associated with global cognitive performance (Table 1), thus no characteristics were included as covariates in additional models.

\section{Subgroup analysis: patients with CAD with major depression}

Significant associations between a greater plasma abundance of PAFs $\mathrm{PC}(O-16: 0 / 2: 0)$ and $\mathrm{PC}(O-18: 0 / 2: 0)$ with poorer global cognitive performance were observed in a subgroup of patients with CAD with major depression (Table 3). Associations with these PAFs and cognition appeared to be driven mainly by relationships with poorer executive function. The lyso-PAF PC(O-16:0/0:0) also demonstrated a significant negative association with global cognitive performance in this subgroup (Table 3). Trends between a greater plasma abundance of PAFs $\mathrm{PC}(\mathrm{O}-$ 18:1/2:0), PC(O-18:6/2:0), and lyso-PAF PC(O-18:1/0:0) and poorer global cognitive performance were detected (Table 3). Conversely, a greater plasma abundance of 
Table 1 Participant characteristics

\begin{tabular}{|c|c|c|c|}
\hline Characteristic & $\begin{array}{l}\text { All CAD patients } \\
(N=24)\end{array}$ & $\begin{array}{l}\text { Major depressed subgroup } \\
\qquad(N=15)\end{array}$ & $\begin{array}{l}\text { Associations with Global Cognition } \\
\qquad(N=24)\end{array}$ \\
\hline Sociodemographic & Value & Value & ( $t$ or $r, P$ value) \\
\hline Age & $60.3(9.4)$ & $59.6(9.2)$ & $-0.01,0.98$ \\
\hline Male (\%) & 70.8 & 73.3 & $-0.29,0.78$ \\
\hline Living alone (\%) & 33.3 & 40.0 & $-0.52,0.61$ \\
\hline White (\%) & 70.8 & 66.7 & $1.67,0.20^{*}$ \\
\hline Years of education & $14.9(3.1)$ & $15.3(3.2)$ & $0.10,0.63$ \\
\hline \multicolumn{4}{|l|}{ Vascular risk factors } \\
\hline Hypertension (\%) & 70.8 & 66.7 & $-0.21,0.84$ \\
\hline Smoking history (\%) & 79.2 & 80.0 & $0.56,0.58$ \\
\hline Years smoked & $27.6(13.4)$ & $25.3(13.7)$ & $-0.09,0.70$ \\
\hline Diabetic (\%) & 41.7 & 40.0 & $0.31,0.76$ \\
\hline Body mass index & $29.8(5.4)$ & $28.5(5.3)$ & $0.11,0.61$ \\
\hline Dyslipidemia (\%) & 79.2 & 86.7 & $0.75,0.46$ \\
\hline Number of vascular risk factors & $3.6(1.5)$ & $3.7(1.5)$ & $0.23,0.29$ \\
\hline $\mathrm{VO}_{2}$ peak $(\mathrm{ml} /(\mathrm{kg} \mathrm{min}))$ & $18.5(4.7)$ & $19.1(4.8)$ & $0.17,0.44$ \\
\hline Systolic blood pressure $(\mathrm{mm} \mathrm{Hg})$ & $121.3(17.9)$ & $123.9(16.9)$ & $-0.33,0.11$ \\
\hline Diastolic blood pressure (mm Hg) & $74.5(10.7)$ & $77.3(12.3)$ & $-0.12,0.57$ \\
\hline \multicolumn{4}{|l|}{ Cardiac history } \\
\hline Percutaneous transluminal coronary angioplasty (\%) & 20.8 & 26.7 & $0.74,0.58^{*}$ \\
\hline Myocardial infarction or ischaemic heart disease (\%) & 50.0 & 46.7 & \\
\hline Coronary artery bypass graft (\%) & 29.2 & 26.7 & \\
\hline Median weeks since event & 14.0 & 14.0 & \\
\hline First episode of CAD (\%) & 70.0 & 60.0 & $1.56,0.13$ \\
\hline \multicolumn{4}{|l|}{ Psychometric } \\
\hline Met criteria for a major depressive episode (\%) & 62.5 & 100.0 & $-0.86,0.40$ \\
\hline History of depression (\%) & 45.8 & 53.3 & $0.59,0.56$ \\
\hline HAM-D score & $12.5(4.8)$ & $14.9(3.8)$ & $-0.20,0.35$ \\
\hline \multicolumn{4}{|l|}{ Concomitant medications (\%) } \\
\hline Antidepressants & 8.3 & 6.7 & $0.64,0.53$ \\
\hline Acetylsalicylic acid & 75.0 & 80.0 & $1.46,0.16$ \\
\hline Platelet inhibitor & 66.7 & 60.0 & $-1.33,0.20$ \\
\hline Statin & 100.0 & 100.0 & - \\
\hline Anti-inflammatory & 4.2 & 6.7 & - \\
\hline Anti-diabetic agents & 37.5 & 33.3 & $0.45,0.66$ \\
\hline
\end{tabular}

$\mathrm{VO}_{2}$, volume of oxygen; HAM-D, Hamilton rating scale for depression; $t$ test and Pearson coefficient summarized associations between participant characteristics and global cognitive performance where appropriate. Data summarized using mean (with standard deviation) unless otherwise stated. *One-way analysis of variance (ANOVA) (with $F$ value) performed for global cognition across ethnicity and cardiac history groups, respectively.

the PAF PC(O-19:5/2:0) was associated with better global cognitive performance in patients with CAD with depression (Table 3). Sample size precluded covariate-adjusted models in this subgroup. Neither $\mathrm{PC}(O-16: 0 / 0: 0)(r=0.17$, $P=0.66), \mathrm{PC}(O-16: 0 / 2: 0)(r=-0.08, P=0.83)$, nor $\mathrm{PC}(O-$ 18:0/2:0) $(r=-0.17, P=0.66)$ were associated with global cognitive performance in patients with CAD without depression $(N=9)$. The PAF PC $(O-19: 5 / 2: 0)(r=-0.73,0.04)$ was significantly associated with poorer global cognitive performance in patients with CAD without depression.

\section{Discussion}

These preliminary findings support the hypothesis that the PAF family of lipid messengers might be associated with cognitive deficits, with strong associations with executive dysfunction driving many of the observed relationships. 
Table 2 Performance ( $z$ scores) on cognitive tasks of the NINDS-CSN cognitive battery

\begin{tabular}{|c|c|c|}
\hline Cognitive task & $\begin{array}{l}\text { All CAD patients }(N=24) z \text { score } \\
\text { (standard deviation) }\end{array}$ & $\begin{array}{c}\text { Major depressed subgroup }(N=15) \text { z score } \\
\text { (standard deviation) }\end{array}$ \\
\hline Executive function & $-0.35(0.70)$ & $-0.36(0.76)$ \\
\hline Animal Naming Task & $-0.28(0.96)$ & $-0.31(0.96)$ \\
\hline FAS/COWAT & $-0.25(0.92)$ & $-0.20(1.06)$ \\
\hline Trail Making Test Part B & $-0.53(0.86)$ & $-0.58(0.93)$ \\
\hline Attention and processing speed & $-0.55(0.78)$ & $-0.64(0.82)$ \\
\hline Trail Making Test Part A & $-0.67(1.00)$ & $-0.81(1.01)$ \\
\hline Digit-Symbol Substitution Test & $-0.43(0.76)$ & $-0.49(0.80)$ \\
\hline Memory & $-0.22(0.95)$ & $-0.37(1.00)$ \\
\hline CVLT, immediate recall (Trials 1 to 5) (verbal) & $-0.51(1.06)$ & $-0.76(1.01)$ \\
\hline CVLT, delayed recall (verbal) & $-0.17(0.93)$ & $-0.30(0.96)$ \\
\hline BVMT, immediate recall (Trials 1 to 3) (visuospatial) & $-0.25(1.39)$ & $-0.35(1.45)$ \\
\hline BVMT, delayed recall (visuospatial) & $-0.02(1.17)$ & $-0.01(1.19)$ \\
\hline Global cognition $z$ score (composite of all sub-domains) & $-0.38(0.62)$ & $-0.46(0.59)$ \\
\hline
\end{tabular}

BVMT, Brief Visuospatial Memory Test; CVLT, California Verbal Learning Test Il; FAS/COWAT, FAS Controlled Word Association Test; NINDS-CSN, National Institute of Neurological Disorders and Stroke-Canadian Stroke Network.

This study also suggests that the relationship between PAFs and cognitive deficits is strongest in patients with CAD with major depression, implicating PAFs as potentially highly relevant to cognitive decline in that subgroup.

Deficits in executive function are predictive of accelerated cognitive decline and are often observed in depressed individuals [31,32]. For example, executive dysfunction is a hallmark feature of depression associated with vascular risk factors in later life [33]. As such, executive dysfunction may be relevant to the increased risk of cognitive decline in those with CAD and, in particular, patients with CAD with depression. In addition to the risk of cognitive decline associated with executive dysfunction in later life, deficits in that domain are associated with poor health outcomes, such as lower quality of life [34], more frequent falls [35], reduced adherence to medications or general health behaviors [36], and in those with depression, reduced effectiveness of antidepressant interventions [37], among many others. Thus, PAFs may be associated with deficits in this clinically important cognitive domain. Our results extend the previous literature, as several of the PAFs found to be associated with cognitive deficits in this study have established mechanistic relationships with neurodegenerative processes. For example, previous preclinical work has identified the PAFs $\mathrm{PC}(O-18: 0 / 2: 0)$ and $\mathrm{PC}(\mathrm{O}-16: 0 / 2: 0)$ as important pathological species associated with pro-apoptotic effects in the central nervous system [18]. Furthermore, $\mathrm{PC}(O-16: 0 / 2: 0)$ and its lyso-PAF precursor, $\mathrm{PC}(O-16: 0 / 0: 0)$, were found to be elevated in the temporal cortex of patients with Alzheimer's disease post-mortem [20], collectively implicating the $\mathrm{PC}(\mathrm{O}-16: 0$ / 2:0) pathway as a potential marker of neurodegeneration.
These results are also consistent with previous literature demonstrating that different PAF species have unique signaling effects both at the PAF receptor (a major conduit for PAF signaling) and independently of the PAF receptor [18]. For example, activation of the PAF receptor by $\mathrm{PC}(\mathrm{O}-16: 0 /$ 2:0) has been shown to be anti-apoptotic, whereas activation by $\mathrm{PC}(O-18: 0 / 2: 0)$ is pro-apoptotic. Additionally, both $\mathrm{PC}(O-16: 0 / 2: 0)$ and $\mathrm{PC}(O-18: 0 / 2: 0)$ have been shown to be pro-apoptotic through different PAF receptor-independent pathways [18]. Thus, each PAF identified in the present study may have a unique signaling potential not only within the periphery, from where it was sampled, but also in the central nervous system, and its effects may be variable depending on whether or not it is activating the PAF receptor. This could explain why the PAF PC $(O-19: 5 / 2: 0)$ was associated with better global cognitive performance in patients with CAD with depression in this study. For example, it is possible that $\mathrm{PC}(O-19: 5 / 2: 0)$ may signal predominantly at the PAF receptor (or predominantly independently of the PAF receptor) and that its signaling effect may be antiapoptotic. It is also possible that $\mathrm{PC}(\mathrm{O}-19: 5 / 2: 0)$ might be an anti-inflammatory member of the PAF family whose role is to counteract the pro-inflammatory effects of the other PAFs. While caution should be exercised when interpreting the differential associations between $\mathrm{PC}(\mathrm{O}-19: 5 / 2: 0)$ and global cognitive performance in patients with and without depression, this PAF may be of mechanistic interest for future study. Unfortunately, the mechanisms by which most of the PAFs identified by this study signal neurodegenerative processes or might be involved in them have yet to be determined. However, the variable negative, neutral, and positive associations between PAFs and cognitive 
Table 3 Pearson correlations between PAFs and global cognitive performance (composite $z$ score) in patients with CAD

\begin{tabular}{|c|c|c|c|c|}
\hline \multirow{2}{*}{$\begin{array}{l}\text { PAF species ( } m / z \\
\text { peak and identities) }\end{array}$} & \multicolumn{2}{|c|}{ All patients $(N=24)$} & \multicolumn{2}{|c|}{ Major depressed subgroup $(N=15)$} \\
\hline & Pearson coefficient $(r)$ & $P$ & Pearson coefficient $(r)$ & $P$ \\
\hline (468.3a) $\mathrm{PC}(0-12: 0 / 2: 0)$ & -0.09 & 0.67 & 0.33 & 0.23 \\
\hline (482.4a) PC(O-13:0/2:0) & 0.17 & 0.44 & 0.19 & 0.49 \\
\hline (496.3a) PC(O-14:0/2:0) & -0.22 & 0.31 & -0.39 & 0.16 \\
\hline (494.3a) PC(O-14:1/2:0) & -0.22 & 0.30 & 0.26 & 0.36 \\
\hline (510.4d) $P C(0-15: 0 / 2: 0)$ & 0.20 & 0.36 & -0.12 & 0.66 \\
\hline (482.4c) PC(O-16:0/0:0) & -0.27 & 0.21 & -0.53 & $0.04^{*}$ \\
\hline (524.4a) PC(O-16:0/2:0) & -0.35 & $0.09^{*}$ & -0.52 & $0.04^{*}$ \\
\hline (522.4a) $\mathrm{PC}(0-16: 1 / 2: 0)$ & 0.06 & 0.78 & -0.03 & 0.92 \\
\hline (520.3a) PC(O-16:2/2:0) & -0.08 & 0.72 & -0.03 & 0.92 \\
\hline (532.3a) $\mathrm{PC}(0-17: 3 / 2: 0)$ & 0.00 & 0.99 & 0.12 & 0.68 \\
\hline (510.4d) $P C(0-18: 0 / 0: 0)$ & -0.08 & 0.73 & -0.04 & 0.89 \\
\hline (552.4e) PC(O-18:0/2:0) & -0.45 & $0.03^{*}$ & -0.59 & $0.02^{*}$ \\
\hline (508.6b) PC(O-18:1/0:0) & 0.22 & 0.31 & 0.45 & $0.09^{*}$ \\
\hline (550.4a) PC(O-18:1/2:0) & -0.37 & $0.08^{*}$ & -0.47 & $0.08^{*}$ \\
\hline (548.4b) PC(O-18:2/2:0) & -0.10 & 0.64 & 0.14 & 0.61 \\
\hline (546.3a) PC(O-18:3/2:0) & -0.15 & 0.50 & 0.19 & 0.50 \\
\hline (544.4a) PC(O-18:4/2:0) & 0.14 & 0.51 & 0.16 & 0.55 \\
\hline (542.3b) PC(O-18:5/2:0) & -0.08 & 0.69 & 0.28 & 0.31 \\
\hline (540.4a) PC(O-18:6/2:0) & -0.40 & $0.05^{*}$ & -0.49 & $0.06^{*}$ \\
\hline (556.4d) PC(O-19:5/2:0) & 0.12 & 0.59 & 0.55 & $0.03^{\dagger}$ \\
\hline (572.3b) PC(O-20:4/2:0) & 0.09 & 0.68 & 0.32 & 0.25 \\
\hline (570.3e) PC(O-20:5/2:0) & -0.29 & 0.16 & 0.03 & 0.92 \\
\hline (568.4a) PC(O-20:6/2:0) & -0.16 & 0.46 & 0.14 & 0.61 \\
\hline
\end{tabular}

Nomenclature adheres to the recent reorganization of ontologies for lipid classes and the curated list of lipid species identified by the Canadian Institutes of Health Research Training Program in Neurodegenerative Lipidomics (CTPNL) teams [28,30]. PC(O-20:4/2:0), for example, defines a lipid species with a phosphocholine polar head group (PC), an ether linkage at the $s n-1$ position ( $O$-) with a chain of 20 carbons and 2 carbons linked by an ester linkage at the sn-2 position, of which the $s n-1$ chain has four degrees of unsaturation (indicated as: 4 ) whereas the sn- 2 chain is characterized by fully saturated acetyl group (referred to by: 0 ). The number preceding species name in brackets refers to $m / z$ of the $[\mathrm{M}+\mathrm{H}]^{+}$ion. Letters distinguish species isobaric with other lipid second messengers in the curated CTPNL phospholipid list. ${ }^{*}$ Significant negative associations $(P \leq 0.05)$; ${ }^{\dagger}$ significant positive associations $(P \leq 0.05)$; $C A D$, coronary artery disease; $P A F$, platelet-activating factor.

performance might suggest roles for specific PAFs in mechanisms of cognitive decline and it may be possible, through lipidomics, to identify important pathways for future investigation.

These preliminary findings are consistent with previous studies demonstrating an interaction between inflammatory messengers and cognitive deficits in populations without dementia. For example, poorer global cognitive performance has previously been associated with greater concentrations of inflammatory cytokines and acute-phase proteins [3]. Inflammatory markers, such as interleukin-6 and C-reactive protein, have also been associated with poorer performance on tests of psychomotor speed and verbal memory in individuals with depression [38-40]. As such, the role of those markers in the relationship between PAFs and cognitive performance in patients with CAD with depression should be addressed in future studies.
This hypothesis-generating study is novel in several aspects: firstly, these findings are the first indication that PAF metabolism might be linked to cognitive deficits in CAD patients, with high relevance to patients with CAD with depression, a group that is at an elevated risk of accelerated cognitive decline [6]. Secondly, most of the available literature on PAFs does not describe PAF species individually, instead classifying PAFs as a type of lipid (that is, a PAF). Only recently has technology permitted a lipidomic approach to identifying unique PAF species $[26,41]$. These findings are the first to characterize individual PAF species in plasma from a clinical sample, adding much needed context to their potentially unique roles as bioactive lipid messengers. Finally, the potential of these preliminary findings is enhanced by the lack of confounding associations between participant characteristics and cognitive outcomes. 


\section{Limitations}

The interpretation of these findings was limited by the small sample size and cross-sectional study design. Moreover, since the study was intended to generate future hypotheses, no adjustment was made for multiple comparisons. As such, it is possible that the associations (or lack thereof) between certain PAFs and cognitive measures might change in a larger sample, revealing the true associations. As the study population consisted only of older patients with CAD, we cannot comment on the potential associations between PAFs and cognitive deficits in those who are medically healthy, those with depression who do not have CAD, or those younger than 45. This study also did not address measures of other cognitive domains (for example, episodic memory) and therefore these findings cannot be generalized across all cognitive domains. Finally, these findings are limited to PAFs derived from alkylacylglycerophosphocholines; however, as mentioned, these are highly abundant PAFs and are therefore appropriate for this exploratory pilot study.

\section{Future directions}

The PAFs in peripheral blood may indicate a systemic state of aberrant lipid metabolism, owing to pathophysiological processes of cognitive decline or may signal across the blood-brain barrier to initiate synthesis of PAFs and other inflammatory mediators in the central nervous system. If our findings are confirmed in a larger sample, PC $(O-18: 0 / 2: 0)$ and $\mathrm{PC}(O-16: 0 / 2: 0)$ might be particularly important PAFs to explore in patients with CAD with depression, considering their consistency of involvement with global and domain-specific cognitive deficits and their established mechanistic relevance to neurodegeneration $[18,20]$. $\mathrm{PC}(O-18: 6 / 2: 0)$ and $\mathrm{PC}(O-19: 5 / 2: 0)$ are newly identified species that represent potential mediators associated with depression-related cognitive deficits in CAD. Should PAFs be confirmed as consistently associated with cognitive deficits, they might serve as clinically useful biomarkers of vulnerability to cognitive decline in patients with CAD. Future studies might clarify the mechanisms by which these PAFs, and others, might mediate neurodegeneration and cognitive decline in patients with $\mathrm{CAD}$, to identify mechanistically relevant therapeutic targets.

In the meantime, these preliminary findings support a role for PAFs as markers of cognitive deficits in CAD patients and invite investigation of this association in larger studies.

\section{Conclusions}

While preliminary, the findings of this study encourage further investigation of PAF species, particularly $\mathrm{PC}(\mathrm{O}-$ 18:0/2:0), as markers of cognitive deficits in CAD patients. As PAFs are emerging as important lipid mediators at the core of neurodegenerative pathology, are elevated in $\mathrm{CAD}$, and are associated with leading mechanisms of cognitive decline associated with depression, these findings are biologically plausible and potentially clinically relevant for patients with CAD with depression. Larger longitudinal studies of PAFs as markers of cognitive decline in CAD patients with and without depression might clarify their role and clinical utility as biomarkers or therapeutic targets.

\section{Abbreviations}

ANOVA: analysis of variance; CAD: coronary artery disease; CTPNL: Canadian Institutes of Health Research Training Program in Neurodegenerative Lipidomics; PAF: Platelet-activating factor.

\section{Competing interests}

The authors declare that they have no competing interests.

\section{Authors' contributions}

GM conceptualized the study, carried out collection, analysis, and interpretation of the data, and drafted the manuscript. NH, SALB, and KLL conceptualized the study, interpreted the data, and revised the manuscript for intellectual content. HX carried out data analysis and revised the manuscript for intellectual content. DF interpreted the data and revised the manuscript for intellectual content. $\mathrm{PIO}$ conceptualized the study and revised the manuscript for intellectual content. All authors gave final approval of the version to be published and agree to be accountable for all aspects of the work.

\section{Acknowledgements}

The authors acknowledge operating funds from the Ontario Mental Health Foundation and the Canadian Institutes of Health Research MOP 89999 to DF and SALB, MOP 114913 to KLL and NH, and a Strategic Training Initiative in Health Research (STIHR) Canadian Institutes of Health Research Training Program in Neurodegenerative Lipidomics (CTPNL) and Institute of Aging TGF 96121 to KL, DF, and SALB. GM and APB received CTPNL graduate scholarships as well as OGS and FRSQ graduate scholarships respectively. HX was supported by a CTPNL and MITACS post-doctoral fellowships.

\section{Author details}

${ }^{1}$ Sunnybrook Research Institute, Sunnybrook Health Sciences Centre, 2075 Bayview Avenue, Room FG08, Toronto, ON M4N 3M5, Canada. ${ }^{2}$ Department of Pharmacology and Toxicology, University of Toronto, Toronto, ON, Canada. ${ }^{3}$ Canadian Institutes of Health Research Training Program in Neurodegenerative Lipidomics, Ottawa, ON, Canada. ${ }^{4}$ Department of Psychiatry, University of Toronto, Toronto, ON, Canada. ${ }^{5}$ Ottawa Institute of Systems Biology and Neural Regeneration Laboratory, Department of Biochemistry, Microbiology and Immunology, University of Ottawa, Ottawa, ON, Canada. ${ }^{6}$ UHN Toronto Rehabilitation Institute, Toronto, ON, Canada.

Received: 30 January 2014 Accepted: 20 June 2014

Published: 4 July 2014

\section{References}

1. Eggermont LHP, de Boer K, Muller M, Jaschke AC, Kamp O, Scherder EJA: Cardiac disease and cognitive impairment: a systematic review. Heart 2012, 98:1334-1340.

2. Roberts RO, Knopman DS, Geda YE, Cha RH, Roger VL, Petersen RC: Coronary heart disease is associated with non-amnestic mild cognitive impairment. Neurobiol Aging 2010, 31:1894-1902.

3. van Exel E, de Craen AJM, Remarque EJ, Gussekloo J, Houx P, Bootsma-van der Wiel A, Frolich M, Macfarlane PW, Blauw GJ, Westendorp RGJ: Interaction of atherosclerosis and inflammation in elderly subjects with poor cognitive function. Neurology 2003, 61:1695-1701.

4. Zheng L, Mack WJ, Chui HC, Heflin L, Mungas D, Reed B, DeCarli C, Weiner MW, Kramer JH: Coronary artery disease is associated with cognitive decline independent of changes on magnetic resonance imaging in cognitively normal elderly adults. J Am Geriatr Soc 2012, 60:499-504.

5. Lipnicki DM, Sachdev PS, Crawford J, Reppermund S, Kochan NA, Trollor JN, Draper B, Slavin MJ, Kang K, Lux O, Mather KA, Brodaty H: Risk factors for 
late-life cognitive decline and variation with age and sex in the Sydney Memory and Ageing Study. PloS ONE 2013, 8:e65841.

6. Freiheit EA, Hogan DB, Eliasziw M, Patten SB, Demchuk AM, Faris $P$, Anderson T, Galbraith D, Parboosingh JS, Ghali WA, Knudtson M, Maxwell CJ: A dynamic view of depressive symptoms and neurocognitive change among patients with coronary artery disease. Arch Gen Psychiatry 2012, 69:244-255

7. Gao Y, Huang C, Zhao K, Ma L, Qiu X, Zhang L, Xiu Y, Chen L, Lu W, Huang C, Tang Y, Xiao Q: Depression as a risk factor for dementia and mild cognitive impairment: a meta-analysis of longitudinal studies. Int J Geriatr Psychiatry 2013, 28:441-449.

8. Swardfager W, Herrmann N, Marzolini S, Oh PI, Saleem M, Shammi P, Kiss A, Cappell J, Lanctot KL: Verbal memory performance and completion of cardiac rehabilitation in patients with coronary artery disease. Psychosom Med 2011, 73:580-587.

9. O'Donnell M, Teo K, Gao P, Anderson C, Sleight P, Dans A, Marzona I, Bosch J, Probstfield J, Yusuf S: Cognitive impairment and risk of cardiovascular events and mortality. Eur Heart J 2012, 33:1777-1786.

10. Dowlati Y, Herrmann N, Swardfager WL, Reim EK, Lanctot KL: Efficacy and tolerability of antidepressants for treatment of depression in coronary artery disease: a meta-analysis. Can J Psychiatry 2010, 55:91-99.

11. Blumenthal JA, Sherwood A, Babyak MA, Watkins LL, Smith PJ, Hoffman BM, O'Hayer CV, Mabe S, Johnson J, Doraiswamy PM, Jiang W, Schocken DD, Hinderliter AL: Exercise and pharmacological treatment of depressive symptoms in patients with coronary heart disease: results From the UPBEAT (Understanding the Prognostic Benefits of Exercise and Antidepressant Therapy) study. J Am Coll Cardiol 2012, 16:1053-1063.

12. Delrieu J, Piau A, Caillaud C, Voisin T, Vellas B: Managing cognitive dysfunction through the continuum of Alzheimer's disease: role of pharmacotherapy. CNS Drugs 2011, 25:213-226.

13. Bennett SAL, Valenzuela N, Xu H, Franko B, Fai S, Figeys D: Using neurolipidomics to identify phospholipid mediators of synaptic (dys) function in Alzheimer's disease. Front Physiol 2013, 4:168.

14. Agostinho P, Cunha RA, Oliveira C: Neuroinflammation, oxidative stress and the pathogenesis of Alzheimer's disease. Curr Pharm Des 2010 $16: 2766-2778$

15. Stapelberg NJC, Neumann DL, Shum DHK, McConnell H, Hamilton-Craig I: A topographical map of the causal network of mechanisms underlying the relationship between major depressive disorder and coronary heart disease. Aust N Z J Psychiatry 2011, 45:351-369.

16. Baune BT, Stuart M, Gilmour A, Wersching H, Heindel W, Arolt V, Berger K: The relationship between subtypes of depression and cardiovascular disease: a systematic review of biological models. Trans/ Psychiatry 2012, 13:e92.

17. Moylan S, Maes M, Wray NR, Berk M: The neuroprogressive nature of major depressive disorder: pathways to disease evolution and resistance, and therapeutic implications. Mol Psychiatry 2013, 18:595-606.

18. Ryan SD, Harris CS, Carswell CL, Baenziger JE, Bennett SAL: Heterogeneity in the $s n-1$ carbon chain of platelet-activating factor glycerophospholipids determines pro- or anti-apoptotic signaling in primary neurons. I Lipid Res 2008, 49:2250-2258.

19. Mazereeuw G, Herrmann N, Bennett SAL, Swardfager W, Xu H, Valenzuela N, Fai S, Lanctôt KL: Platelet activating factors in depression and coronary artery disease: a potential biomarker related to inflammatory mechanisms and neurodegeneration. Neurosci Biobehav Rev 2013, 37:1611-1621.

20. Ryan SD, Whitehead SN, Swayne LA, Moffat TC, Hou W, Ethier M, Bourgeois AJG, Rashidian J, Blanchard AP, Fraser PE, Park DS, Figeys D, Bennett SAL: Amyloid- $\beta 42$ signals tau hyperphosphorylation and compromises neuronal viability by disrupting alkylacylglycerophosphocholine metabolism. Proc Natl Acad Sci USA 2009, 106:20936-20941.

21. Hachinski V, ladecola C, Petersen RC, Breteler MM, Nyenhuis DL, Black SE, Powers WJ, DeCarli C, Merino JG, Kalaria RN, Vinters HV, Holtzman DM, Rosenberg GA, Wallin A, Dichgans M, Marler JR, Leblanc GG: National Institute of Neurological Disorders and Stroke - Canadian Stroke Network vascular cognitive impairment harmonization standards. Stroke 2006, 37:2220-2241. A published erratum appears in Stroke 2007, 38(3):1118.

22. Benedict R: Brief Visuospatial Memory Test-Revised: Professional Manual. Lutz, FL: Psychological Assessment Resources, Inc:; 1997.

23. Hamilton A: A rating scale for depression. I Neurol Neurosurg Psychiatry 1960, 23:56-62.
24. First MB, Spitzer RL, Gibbon M, Williams JBW: Structured Clinical Interview for DSM-IV diagnoses (SCID): Clinician and Research Versions. New York, NY: Biometrics Research Department, Columbia University; 1996

25. Bligh EC, Dyer WJ: A rapid method of lipid extraction and purification. Can J Biochem 1959, 37:911-917.

26. Xu H, Valenzuela N, Fai S, Figeys D, Bennett SAL: Targeted lipidomics: advances in profiling lysophosphocholine and platelet activating factor second messengers. FEBS J 2013, 280:5652-5667.

27. Whitehead SN, Hou W, Ethier M, Smith JC, Bourgeois A, Denis R, Bennett SAL, Figeys D: Identification and quantitation of changes in the platelet activating factor family of glycerophospholipids over the course of neuronal differentiation by high-performance liquid chromatography electrospray ionization tandem mass spectrometry. Anal Chem 2007, 79:8539-8548

28. Blanchard AP, McDowell GSV, Valenzuela N, Xu H, Gelbard S, Bertrand M Slater GW, Figeys D, Fai S, Bennett SAL: Visualization and Phospholipid Identification (VaLID): online integrated search engine capable of identifying and visualizing glycerophospholipids with given mass. Bioinformatics 2013, 29:284-285.

29. Tang C-H, Tsao P-N, Chen C-Y, Shiao M-S, Wang W-H, Lin C-Y: Glycerophosphocholine molecular species profiling in the biological tissue using UPLC/MS/MS. J Chromatogr B Analyt Technol Biomed Life Sci 2011, 879:2095-2106.

30. Fahy E, Cotter D, Sud M, Subramaniam S: Lipid classification, structures and tools. Biochim Biophys Acta 1811, 2011:637-647.

31. Blacker D, Lee H, Muzikansky A, Martin EC, Tanzi R, McArdle JJ, Moss M, Albert M: Neuropsychological measures in normal individuals that predict subsequent cognitive decline. Arch Neurol 2007, 64:862-871.

32. McDermott LM, Ebmeier KP: A meta-analysis of depression severity and cognitive function. J Affect Disord 2009, 119:1-8.

33. Alexopoulos GS: The vascular depression hypothesis: 10 years later. Biol Psychiatry 2006, 60:1304-1305.

34. Brookes RL, Herbert V, Paul S, Hannesdottir K, Markus HS, Morris RG: Executive dysfunction, awareness deficits and quality of life in patients with cerebral small vessel disease: a structural equation model. Neuropsychology 2014, 28:247-253.

35. Kearney FC, Harwood RH, Gladman JRF, Lincoln N, Masud T: The relationship between executive function and falls and gait abnormalities in older adults: a systematic review. Dement Geriatr Cogn Disord 2013, 36:20-35.

36. Stoehr GP, Lu S-Y, Lavery L, Bilt JV, Saxton JA, Chang C-CH, Ganguli M: Factors associated with adherence to medication regimens in older primary care patients: the Steel Valley Seniors Survey. Am J Geriatr Pharmacother 2008, 6:255-263.

37. Taylor WD, Aizenstein HJ, Alexopoulos GS: The vascular depression hypothesis: mechanisms linking vascular disease with depression. Mol Psychiatry 2013, 18:963-974.

38. Chang HH, Lee IH, Gean PW, Lee S-Y, Chi MH, Yang YK, Lu R-B, Chen PS: Treatment response and cognitive impairment in major depression: association with C-reactive protein. Brain Behav Immun 2012, 26:90-95

39. Grassi-Oliveira R, Bauer ME, Pezzi JC, Teixeira AL, Brietzke E: Interleukin-6 and verbal memory in recurrent major depressive disorder. Neuro Endocrinol Lett 2011, 32:540-544.

40. Krogh J, Benros ME, Jørgensen MB, Vesterager L, Elfving B, Nordentoft M: The association between depressive symptoms, cognitive function, and inflammation in major depression. Brain Behav Immun 2014, 35:70-76.

41. Bou Khalil M, Hou W, Zhou H, Elisma F, Swayne LA, Blanchard AP, Yao Z, Bennett SAL, Figeys D: Lipidomics era: accomplishments and challenges. Mass Spectrom Rev 2010, 29:877-929.

doi:10.1186/1742-2094-11-119

Cite this article as: Mazereeuw et al:: Platelet-activating factors are associated with cognitive deficits in depressed coronary artery disease patients: a hypothesis-generating study. Journal of Neuroinflammation 2014 11:119. 